\title{
Correlation between Overactive Bladder Syndrome and Obsessive Compulsive Disorder in Women
}

\author{
Keun-Soo Ahn, Hyun-Pyo Hong, Hyuk-Jung Kweon*, Ah-Leum Ahn, Eun-Jung Oh, Jae-Kyung Choi, Dong-Yung Cho \\ Department of Family Medicine, KonKuk University Medical Center, Seoul, Korea
}

Background: Overactive bladder syndrome is characterized by urinary urgency, usually accompanied by Frequent urination and nocturia, with or without urgent urinary incontinence. There must be the absence of causative infection or pathological conditions. Overactive bladder syndrome is related to mental disorders, particularly depression and anxiety. However, obsessive-compulsive symptoms are investigated much less frequently. The purpose of the present study was thus to assess obsessive-compulsive symptoms in overactive bladder syndrome patients.

Methods: Fifty-seven women patients with overactive bladder syndrome and fifty-seven women without it (age matched control group) were prospectively enrolled. They completed the overactive bladder syndrome-validated 8-question screener and the Korean version of the Maudsley Obsessional-Compulsive Inventory Questionnaire at the same time they visited the clinic. Patients were compared with controls on the Korean version of the Maudsley Obsessional-Compulsive Inventory Questionnaire and its checking, tidiness, doubting, and fear of contamination components.

Results: Patients showed more obsessive traits than controls on the Korean version of the Maudsley ObsessionalCompulsive Inventory Questionnaire total score $(\mathrm{P}=0.006)$ and on the checking subscale $(\mathrm{P}=0.001)$. Odds ratio for the overactive bladder syndrome group's obsessive-compulsive symptoms traits (score $\geq 14$ ) was $5.47(\mathrm{P}=0.001)$. The Korean version of the Maudsley Obsessional-Compulsive Inventory Questionnaire total score was associated with the overactive bladder syndrome-validated 8-question screener score in patients $(\mathrm{P}=0.03)$.

Conclusion: Obsessive-compulsive symptoms may constitute an important aspect of the psychiatric profile of overactive bladder syndrome patients. The severity of obsessive-compulsive symptoms seems to be related to the degree of the overactive bladder syndrome severity. Clinicians may consider screening women with overactive bladder syndrome for obsessive-compulsive symptoms.

Keywords: Overactive Bladder; Obsessive Compulsive Disorder; Questionnaire; Women 


\section{INTRODUCTION}

According to the International Continence Society, overactive bladder syndrome $(\mathrm{OAB})$ is characterized by urinary urgency, usually accompanied by frequency and nocturia, with or without urgency urinary incontinence in the absence of causative infection or pathological conditions. ${ }^{1)}$

A population-based survey conducted by Milsom et al. ${ }^{2)}$ in 6 countries estimated overall prevalence of $\mathrm{OAB}$ in Europe to be $16.6 \%$ (15.6\% and $17.4 \%$ for men and women). A report from the European Prospective Investigation into Cancer and Nutrition (EPIC) study estimated the overall prevalence of OAB in Europe and Canada to be $12.8 \%$ among women and $10.8 \%$ among men. ${ }^{3)}$ In the National Overactive Bladder Evaluation survey, conducted in the United States, overall prevalence of OAB was $16.9 \%$ in women and $16.0 \%$ in men. ${ }^{4)}$ The Korean EPIC study, conducted in 2006, reported the overall prevalence of OAB to be $12.2 \%$ ( $10.0 \%$ of men and $14.3 \%$ of women $)^{5)}$

$\mathrm{OAB}$ is a highly prevalent disorder with an unknown etiology. Its symptoms impair various aspects of a patient's physical and mental daily life, and it is considered a large socioeconomic burden. ${ }^{4}$

There are hypotheses that mental health and urological conditions may share common neuropharmacological correlates like corticotropin releasing factor. ${ }^{6}$ Some serotonergic pathways are believed to be related to Obsessive Compulsive Disorder (OCD), anxiety disorders, and depression. ${ }^{7,8)}$ The clinical use of serotonin transport inhibitors in the treatment of OCD is well known. ${ }^{9)}$ There is support that OCD is related to other anxiety and affective disorders. Studies have demonstrated the relationship of $\mathrm{OAB}$ with depression and anxiety. ${ }^{10,11)}$ Relationships between $\mathrm{OAB}$ and OCD have been suggested in one study. ${ }^{12)}$ However, it did not administer OCD specific questionnaires. No previous study has been conducted in South Korea about this topic yet. The aim of the present study was to systematically investigate obsessive-compulsive traits in $\mathrm{OAB}$ patients.

\section{METHODS}

\section{Study Population}

The patient group was 100 women over 20 years of age in a rural area, South Korea, who visited the department of urology clinic of Konkuk University Medical Center, Chungju Hospital as an outpatient between August 2008 and November 2010. They were all diagnosed with $\mathrm{OAB}$ by a urology specialist, however some patients with $\geq 8 \mathrm{OAB}$-validated 8-question screener (OAB-V8) scores $(n=30)$, who had psychological history (such as major depressive disorder) $(n=3)$ were excluded, as were participants with incomplete questionnaire $(n=10)$. Therefore, a total of 57 women were included in the final analysis. The survey involved face-to-face interviews, with fully informed consent.

The control group participants were 300 women over 20 years of age from the same community, who visited the department of family medicine clinic of Konkuk University Medical Center, Chungju Hospital as an outpatient between October 2008 and December 2010. None of the participants sought treatment for OAB but some patients with $\geq 8$ of OAB-V8 scores ( $n=50$ ), who had psychological history (such as major depressive disorder) $(n=20)$ were excluded, as were participants with incomplete questionnaire $(n=10)$. Participants were selected randomly and matched by age. The final analysis included 57 subjects. The survey involved face-to-face interviews, with fully informed consent.

\section{Study Procedure}

According to data from validation studies, the Korean version of the OAB-V8 was validated, ${ }^{13,14)}$ and the Korean version of the Maudsley Obsessional-Compulsive Inventory (MOCI-K) was validated in clinical samples ${ }^{15,16)}$ and in nonclinical samples. ${ }^{15,17,18)}$ These condition specific questionnaires were used for this analysis. This observational study was conducted on patients with $\mathrm{OAB}$ and designed only for psychometric evaluation using the translated OAB-V8 and MOCI-K. Therefore, no investigational medications or procedures were used in the study; only the questionnaires were administered.

OAB-V8 questionnaires included 8 questions on evidence of LUTS (lower urinary tract symptoms). ${ }^{13,14)}$ Responses were answered on a 6-point Symptom Bother scale ranging from 0 (not at all) to 5 (a very great deal). In order to assess obsessive-compulsive symptoms (OCS), we administered the MOCI-K, a patient-completed questionnaire of most common OCS. The inventory includes both affirmative and negative sentences. The MOCI-K is composed of 30 questions, which are classified into five factions: checking ( 6 items), tidiness ( 6 items), doubting (6 items), fear of contamination (6 items), ruminating (2 items), and miscellaneous (4 items). Answers ranged from 0 (no) to 1 (yes). ${ }^{18)}$ In this study, patients were considered to have $\mathrm{OAB}$ if their OAB-V8 scores were $\geq 8,{ }^{14)}$ OCD traits if their MOCI-K scores were $\geq 14{ }^{18)}$ MOCI-K was shown to have good internal consistency, good test-retest reliability, and good discriminant and convergent validity. ${ }^{18)}$

\section{Statistical Analysis}

Internal consistency of the 4 subscales of MOCI and the entire test were found to be satisfactory in both the control group and the patient group yielding Cronbach $\alpha$ coefficients between 0.69 and 0.82 . Continuous variables were compared across groups with Student t-tests or, in the case of non-normally distributed variables, with nonparametric tests (two-tailed significance level of $\mathrm{P}=0.05)$. The Pearson correlation coefficient $(\mathrm{r})$ was used to determine whether OAB-V8 score was associated with 
MOCI-K score for each group. Partial correlations were run to control for potentially secondary effects. Two-sided P-values less than .05 were statistically significant. The relationship between the OAB and OCD traits (MOCI-K score $\geq 14$ ) were analyzed using logistic regression analysis. Wald chi-square and corresponding P-values were used to describe the relationship of $\mathrm{OAB}$ with $\mathrm{OCD}$ traits after adjusting for age differences in the 2 groups. Odds ratios were calculated for the likelihood of having OCD traits given the presence of OAB. Statistical analysis was performed with IBM SPSS ver. 20.0 (IBM Co., Armonk, NY, USA) software.

\section{RESULTS}

\section{Comparison of Baseline Characteristics of the Subjects}

A total of 114 participants were included (patient group of 57 and control group of 57). All of the participants were Asian women. Table 1 shows the characteristics of all participants and includes age, OAB V8 scores, and MOCI-K scores. The ages of the patient group ranged from 20 to 86 years with a mean age of 50.65 years (standard deviation [SD] 14.72). The highest proportion of age range was $45-54(n=15,26.32 \%)$; the lowest proportion of age range was $<25(n=1,1.75 \%)$. The mean OAB-V8

Table 1. Baseline characteristics of the subjects

\begin{tabular}{ccccc}
\hline Characteristic & $\begin{array}{c}\text { OAB } \\
(n=57)\end{array}$ & $\begin{array}{c}\text { Controls } \\
(\mathrm{n}=57)\end{array}$ & $\begin{array}{c}\text { Mean } \\
\text { difference }\end{array}$ & P-value \\
\hline Age (y) & $50.65 \pm 14.72$ & $50.09 \pm 14.47$ & -0.56 & $0.838^{*}$ \\
$<25$ & $1(1.75)$ & $1(1.75)$ & & \\
$25-34$ & $9(15.79)$ & $9(15.79)$ & & \\
$35-44$ & $11(19.30)$ & $12(20.05)$ & & \\
$45-54$ & $15(26.32)$ & $14(24.57)$ & & \\
$55-64$ & $11(19.30)$ & $10(17.55)$ & & \\
$65-74$ & $6(10.53)$ & $7(12.28)$ & & $0.000^{\dagger}$ \\
$\geq 75$ & $4(7.02)$ & $4(7.02)$ & & $0.006^{\star}$ \\
0AB-V8 & $19.91 \pm 10.14$ & $1.30 \pm 1.85$ & 18.61 & \\
M0Cl-K score & $10.88 \pm 6.59$ & $8.02 \pm 4.05$ & 2.86 & \\
$0-13$ & $36(63.2)$ & $51(89.47)$ & & \\
14-30 & $21(36.8)$ & $6(10.53)$ & &
\end{tabular}

Values are presented as mean \pm standard deviation or number (\%).

$O A B$, overactive bladder syndrome; $O A B-V 8, O A B$-validated 8-question screener; $\mathrm{MOCl}-\mathrm{K}$, Korean version of Maudsley obsessional-compulsive inventory.

*Analyzed using Student unpaired t-test. ${ }^{\dagger}$ Analyzed using Mann-Whitney test.

Table 2. Comparison between $\mathrm{OAB}$ and control groups in $\mathrm{MOCl}-\mathrm{K}$ questionnaires

\begin{tabular}{lcccc}
\hline Variable & $\begin{array}{c}\mathrm{OAB} \\
(\mathrm{n}=57)\end{array}$ & $\begin{array}{c}\text { Control } \\
(\mathrm{n}=57)\end{array}$ & $\begin{array}{c}\text { Mean } \\
\text { difference }\end{array}$ & P-value \\
\hline MOCl-K total score & $10.88 \pm 6.59$ & $8.02 \pm 4.05$ & 2.86 & $0.006^{\star}$ \\
Checking score & $2.88 \pm 1.83$ & $1.84 \pm 1.41$ & 1.04 & $0.002^{\dagger}$ \\
Tidiness score & $1.28 \pm 1.46$ & $0.86 \pm 0.99$ & 0.42 & $0.186^{\dagger}$ \\
Doubting score & $2.70 \pm 1.96$ & $2.32 \pm 1.42$ & 0.36 & $0.383^{\dagger}$ \\
Fear of contamination score & $1.05 \pm 1.14$ & $1.18 \pm 1.23$ & 0.13 & $0.694^{\dagger}$
\end{tabular}

Values are presented as mean \pm standard deviation.

$\mathrm{OAB}$, overactive bladder syndrome; $\mathrm{MOCl}-\mathrm{K}$, Korean version of Maudsley ObsessionalCompulsive Inventory.

*Analyzed using Student unpaired t-test. ${ }^{\dagger}$ Analyzed using Mann-Whitney test. score \pm SD was $19.91 \pm 10.14$. The mean MOCI-K score \pm SD was $10.88 \pm 6.59$.

The control group age ranged from 20 to 79 years with a mean age of $50.09 \pm 14.47$. The highest proportion of age range of age was $45-54(n=14,24.57 \%)$; the lowest proportion age range was $<25(\mathrm{n}=1,1.67 \%)$. The mean OAB-V8 score was $1.30 \pm 1.85$. The Mean MOCI-K score was $8.02 \pm 4.05$. With the exception of the mean OAB-V8 score and MOCI-K score, there were no significant differences between the two groups. No one from either group had other known psychological problems. As predicted, patients showed significantly more obsessive traits than healthy controls on MOCI total score (mean difference 2.86, Pvalue $=0.006$ ) and in the checking subscale (mean difference 1.04, P-value $=0.001)$. A total of $21(36.8 \%)$ of patients had OCD traits (MOCI-K score $\geq 14$ ). A significant increase in OCD traits was noted in the OAB group compared to controls (Table 2).

\section{Evaluation of the Correlation between Overactive Bladder Syndrome-Validated 8-Question Screener Score and Korean Version of the Maudsley Obsessional-Compulsive Inventory Score}

Following these results, we performed additional analyses to look at the correlation between OAB-V8 score and MOCI-K score in more detail. A logistic regression analysis revealed that female patients with $\mathrm{OAB}$ were more likely to have OCD traits than non-OCD control group participants after adjusting for age (odds ratio, 5.47; $\mathrm{P}=0.001$ ) (Table 3). Pearson correlations between OAB-V8 score and MOCI-K were determined for the OCD patient group and control group. Among them, the OCD

Table 3. Relationship between $\mathrm{OAB}$ and $\mathrm{OCD}$ traits $(\mathrm{MOCl}-\mathrm{K} \geq 14)$ adjusted for age

\begin{tabular}{cccc}
\hline & Wald chi-square & P-value & OR (95\% confidence interval) \\
\hline OAB & 10.82 & 0.001 & $5.47(1.99-15.04)$ \\
\hline
\end{tabular}

Chi-square and ORs are from logistic models and include age and $\mathrm{OAB}$ as the independent factors. The $\mathrm{OR}$ is the odds of having OCD traits ( $\mathrm{MOCl}-\mathrm{K} \geq 14$ ) for patients with $O A B$ versus those without, after adjusting for the age.

$\mathrm{OAB}$, overactive bladder syndrome; OCD, obsessive-compulsive disorder; $\mathrm{MOCl}-\mathrm{K}$ Korean version of Maudsley Obsessional-Compulsive Inventory; OR, odds ratio.

Table 4. Correlation between $\mathrm{OAB}-\mathrm{V} 8$ and MOCl-K (total and subscales) adjusted for age

\begin{tabular}{lccccc}
\hline & \multicolumn{2}{c}{ OAB group } & & \multicolumn{2}{c}{ Control group } \\
\cline { 2 - 3 } \cline { 5 - 6 } & $\begin{array}{l}\text { Partial correlation } \\
\text { coefficient }(r)\end{array}$ & P-value $^{*}$ & & $\begin{array}{c}\text { Partial correlation } \\
\text { coefficient }(r)\end{array}$ & P-value* \\
\hline MOCl-K total score & 0.393 & 0.003 & & 0.176 & 0.195 \\
Checking score & 0.355 & 0.001 & & 0.238 & 0.078 \\
Tidiness score & 0.388 & 0.003 & & -0.095 & 0.486 \\
Doubting score & 0.255 & 0.058 & & 0.207 & 0.126 \\
Fear of contamination & 0.222 & 0.100 & & 0.068 & 0.529 \\
$\quad$ score & & & & & \\
\hline
\end{tabular}

OAB-V8, OAB-validated 8-question screener; MOCl-K, Korean version of the Maudsley Obsessional-Compulsive Inventory.

*Analyzed using Pearson correlation test after adjusting for age. 

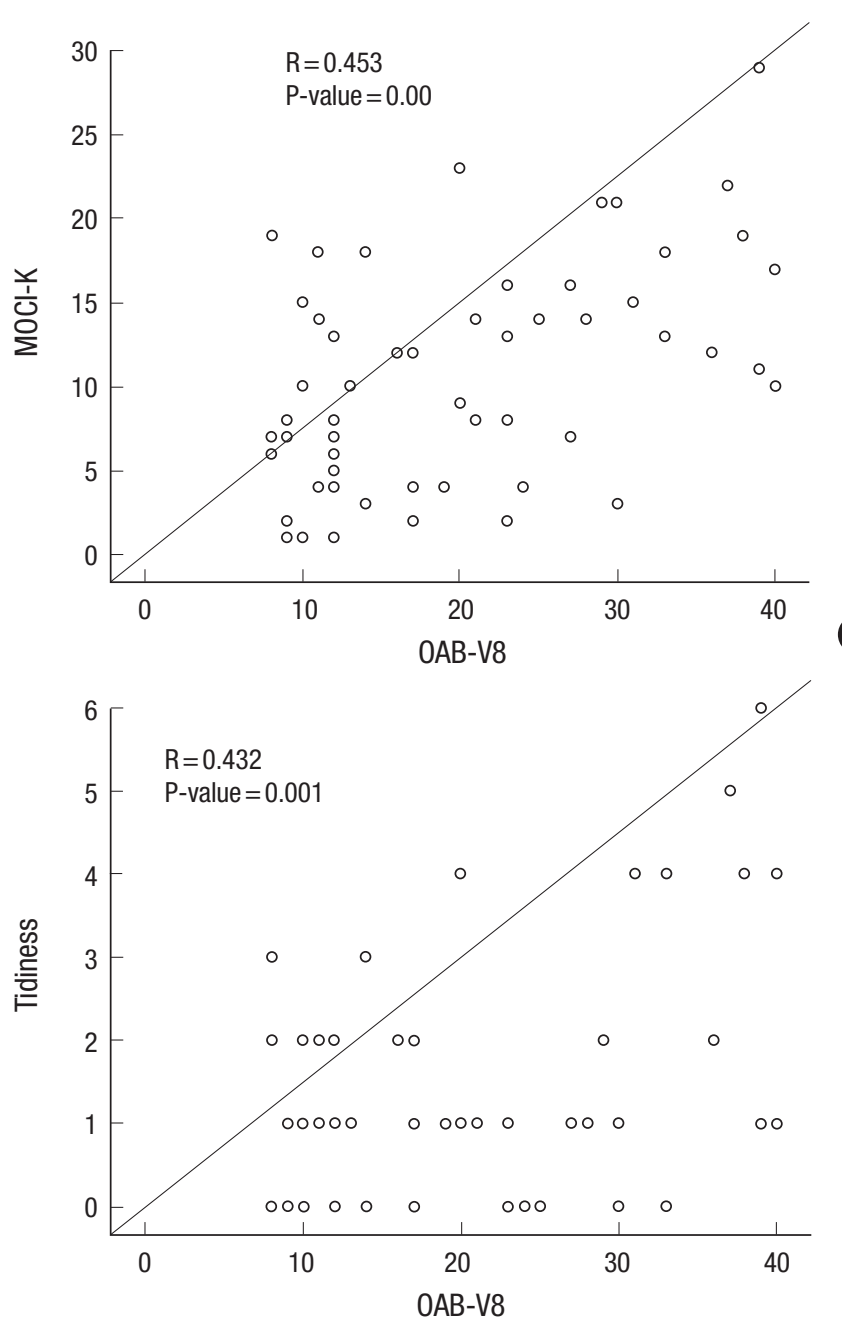

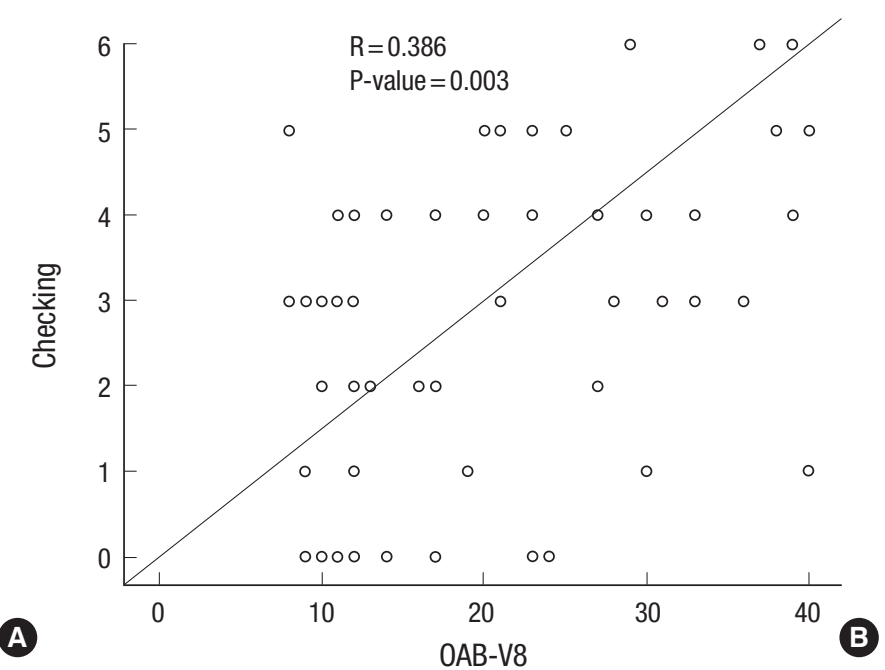

Figure 1. Correlation between $\mathrm{OAB}-\mathrm{V} 8$ and $\mathrm{MOCl}-\mathrm{K}$ (total and subscales) in $O A B$ patient group (unadjusted). These scatter plots manifest the result of Pearson correlation test that was conducted to analyze correlation between OAB-V8 and MOCl-K in OAB patient group. (A) MOCl-K total and OAB-V8. (B) Checking subscale and OAB-V8. (C) Tidiness subscale and OAB-V8. OAB-V8, $\mathrm{OAB}$-validated 8-question screener; MOCl-K, Korean version of the Maudsley Obsessional-Compulsive Inventory. *Analyzed using Pearson correlation test. patient group showed the strongest correlation between $\mathrm{OAB}$ V8 score and MOCI-K score $(\mathrm{R}=0.393, \mathrm{P}=0.003)$ after controlling for age. In contrast, the control group showed no significant correlation between OAB-V8 score and MOCI-K score $(\mathrm{R}=0.18$, $\mathrm{P}=0.195)$. Furthermore, we found significant correlations of MOCI-K with the parameters loaded primarily on the tidiness subscale and secondarily on the checking subscale. Tidiness ( $\mathrm{R}=0.388, \mathrm{P}=0.003)$ and checking $(\mathrm{R}=0.355, \mathrm{P}=0.001)$ subscales correlated with levels of OAB-V8 after controlling for age (Table 4, Figure 1).

\section{DISCUSSION}

Our results suggest that OCS constitutes an important aspect of the psychiatric profile of $\mathrm{OAB}$ patients. In this study, we showed that MOCI-K scores were higher in the patient group, and women with $\mathrm{OAB}$ were more likely to have $\mathrm{OCD}$ traits than non-OAB control group. In addition, in the OCD patient group, the OABV8 scales correlated relatively closely with the MOCI-K scales. These results support the relationship between OAB symptoms and OCD that was observed in the previous study conducted by Hsiao et al., ${ }^{12)}$ where they found higher levels of somatic complaints and OCS in OAB women. However, they administered the Brief Symptom Rating Scale that included all of the following dimensions of psychopathology: somatic symptoms, OCS, inter-personal sensitivity, depressive symptoms, anxiety symptoms, hostility, phobic-anxiety, and paranoid tendency. With this broad ranged scale, they may have investigated other aspects of psychological distress, because the scale was not specific to OCD. In contrast, our study used MOCI-K, which is more specific to OCD. This is a huge difference from previous studies. We tested 57 patients, which is not a small sample for psychometric evaluation. The patient group was not only diagnosed as OAB by a urology specialist, but also scored 8 or more on the OAB-V8 questionnaire. This means there is very little possibility that they are not $\mathrm{OAB}$ patients. While it is not clear if OCD is a causal factor for OAB symptoms or results from having these bladder symptoms, the relationship between OCD and $\mathrm{OAB}$ warrants further exploration.

We recognize that the present study has several limitations. 
First, it is still possible that some patients with psychological diagnoses were included, though we excluded ones with other psychological history. This is possible because individuals with OCD frequently experience additional mental problems such as anxiety disorders and affective disorders. ${ }^{19)}$ In one study, the MOCI was found to discriminate between obsessive patients and patients without OCD. Patients with anorexia nervosa and anxiety disorders, failed to discriminate OCD patients from depressive patients. ${ }^{20)}$ Future studies that include the Korean version of Beck's Depression Inventory and the Korean version of Beck's Anxiety Inventory would be able to exclude these highly co-morbid psychological disorders effectively. Second, we did not collect patient's detailed characteristics at the time of the survey such as diet habit, smoking history, and alcohol consumption. However, other studies which have studied the relationship between $\mathrm{OAB}$ and $\mathrm{OCD}^{12)}$ and Parkinson's disease and $\mathrm{OCD}^{21)}$ also did not consider anything as confounders except for co-morbid psychological disorders. We also did not regard those factors as confounders. Third, we only studied women, since we did not have a large enough sample of men. Fourth, since this study is observational and cross-sectional, we could not see the cause and effect relationship between these two disorders. Thus, we suggest that future studies use an experimental and longitudinal design to figure out the relationship between OCD and OAB. With these methods, future researchers can exam OAB treatment related to MOCI score improvement. Fifth, a relatively low proportion of our control participants had OCD traits. Two studies which used MOCI scores as a method reported control group's scores ranging from 4 to $6 ;^{21,22)}$ and one study which validated MOCI score had 34 scoring items and a mean of $7.55,{ }^{23)}$ which was less than ours, despite that they included 4 more subscale questionnaire items than ours. As noted above, we have excluded all persons with other mental problems from both groups. Therefore, theoretically we expected our control group would have a lower MOCI score than normal. Considering that our control group has a higher mean MOCI-K score than others, there is very little possibility that selection bias has influenced our results. Lastly, we used only the MOCI-K to examine OCS. Patients with OAB presented significantly more self-reported OCS than controls in MOCI-K questionnaires, but none of the patients were diagnosed as having obsessive-compulsive disorder according to DSM-V criteria; and no one was receiving psychopharmacological treatment for obsessive-compulsive disorder symptomatology. Although the MOCI scales are widely accepted as descriptive self-estimation scales for OCS, no diagnostic value was given. However, we can quickly complete self-reported measures without challenging participants' patience, and they may feel more comfortable completing them alone rather than engaging in a conversation with the clinician. Since OCD patients are commonly uncommunicative about their problems, ${ }^{24)}$ the use of self-reported measures may be preferable to guard against the underreporting of OCS symptoms that is often observed during a clinician-administered interview. Moreover, self-reported inventories are free of the bias caused by personal experience, so it is more objective. Furthermore, one major advantage of MOCI is that it examines various types of OCS by means of an internal control of items with repeated content.

In conclusion, we found that $\mathrm{OAB}$ and $\mathrm{OCD}$ have a positive correlation in women. In addition, women with $\mathrm{OAB}$ will have more possibility to have OCD traits than women who do not. These findings suggest that for women with $\mathrm{OAB}$, clinicians may consider screening for OCD, and when appropriate, discuss psychiatrist referral.

\section{CONFLICT OF INTEREST}

No potential conflict of interest relevant to this article was reported.

\section{REFERENCES}

1. Abrams P, Cardozo L, Fall M, Griffiths D, Rosier P, Ulmsten U, et al. The standardisation of terminology in lower urinary tract function: report from the standardisation sub-committee of the International Continence Society. Urology 2003;61:37-49.

2. Milsom I, Abrams P, Cardozo L, Roberts RG, Thuroff J, Wein AJ. How widespread are the symptoms of an overactive bladder and how are they managed?: a population-based prevalence study. BJU Int 2001; 87:760-6.

3. Irwin DE, Milsom I, Hunskaar S, Reilly K, Kopp Z, Herschorn S, et al. Population-based survey of urinary incontinence, overactive bladder, and other lower urinary tract symptoms in five countries: results of the EPIC study. Eur Urol 2006;50:1306-14.

4. Stewart WF, Van Rooyen JB, Cundiff GW, Abrams P, Herzog AR, Corey $\mathrm{R}$, et al. Prevalence and burden of overactive bladder in the United States. World J Urol 2003;20:327-36.

5. Lee YS, Lee KS, Jung JH, Han DH, Oh SJ, Seo JT, et al. Prevalence of overactive bladder, urinary incontinence, and lower urinary tract symptoms: results of Korean EPIC study. World J Urol 2011;29:185-90.

6. Klausner AP, Steers WD. Corticotropin releasing factor: a mediator of emotional influences on bladder function. J Urol 2004;172(6 Pt 2):2570-3.

7. Insel TR, Mueller EA, Alterman I, Linnoila M, Murphy DL. Obsessivecompulsive disorder and serotonin: is there a connection? Biol Psychiatry 1985;20:1174-88.

8. Hu XZ, Lipsky RH, Zhu G, Akhtar LA, Taubman J, Greenberg BD, et al. Serotonin transporter promoter gain-of-function genotypes are linked to obsessive-compulsive disorder. Am J Hum Genet 2006;78:815-26.

9. Apter A, Ratzoni G, King RA, Weizman A, Iancu I, Binder M, et al. Fluvoxamine open-label treatment of adolescent inpatients with obsessive-compulsive disorder or depression. J Am Acad Child Adolesc Psychiatry 1994;33:342-8.

10. Milsom I, Kaplan SA, Coyne KS, Sexton CC, Kopp ZS. Effect of bothersome overactive bladder symptoms on health-related quality of life, 
anxiety, depression, and treatment seeking in the United States: results from EpiLUTS. Urology 2012;80:90-6.

11. Yoo ES, Kim BS, Kim DY, Oh SJ, Kim JC. The impact of overactive bladder on health-related quality of life, sexual life and psychological health in Korea. Int Neurourol J 2011;15:143-51.

12. Hsiao SM, Liao SC, Chen CH, Chang TC, Lin HH. Psychometric assessment of female overactive bladder syndrome and antimuscarinics-related effects. Maturiatas 2014;79:428-34.

13. Acquadro C, Kopp Z, Coyne KS, Corcos J, Tubaro A, Choo MS, et al. Translating overactive bladder questionnaires in 14 languages. Urology 2006;67:536-40.

14. Coyne KS, Zyczynski T, Margolis MK, Elinoff V, Roberts RG. Validation of an overactive bladder awareness tool for use in primary care settings. Adv Ther 2005;22:381-94.

15. Sternberger LG, Burns GL. Compulsive Activity Checklist and the Maudsley Obsessional-Compulsive Inventory: psychometric properties of two measures of obsessive-compulsive disorder. Behav Ther 1991;21: 117-27.

16. Lim JS, Kim SJ, Jeon WT, Cha KR, Park JH, Kim CH. Reliability and validity of the Korean version of Obsessive-Compulsive Inventory-Revised in a non-clinical sample. Yonsei Med J 2008;49:909-16.

17. Sternberger LG, Burns GL. Maudsley Obsessional-Compulsive Inven- tory: obsessions and compulsions in a nonclinical sample. Behav Res Ther 1990;28:337-40.

18. Min BB, Won HT. Reliability and validity of the Korean translations of Maudsley obsessional-compulsive inventory and Padua inventory. Korean J Clin Psychol 1999;18:163-82.

19. Nestadt G, Samuels J, Riddle MA, Liang KY, Bienvenu OJ, Hoehn-Saric $\mathrm{R}$, et al. The relationship between obsessive-compulsive disorder and anxiety and affective disorders: results from the Johns Hopkins OCD Family Study. Psychol Med 2001;31:481-7.

20. Emmelkamp PM, Kraaijkamp HJ, van den Hout MA. Assessment of obsessive-compulsive disorder. Behav Modif 1999;23:269-79.

21. Alegret M, Junque C, Valldeoriola F, Vendrell P, Marti MJ, Tolosa E. Obsessive-compulsive symptoms in Parkinson's disease. J Neurol Neurosurg Psychiatry 2001;70:394-6.

22. Stoylen IJ, Larsen S, Kvale G. The Maudsley Obsessional-Compulsive Inventory and OCD in a Norwegian nonclinical sample. Scand J Psychol 2000;41:283-6.

23. Woods CM. Factor analysis of scales composed of binary items: illustration with the Maudsley Obsessional Compulsive Inventory. J Psychopathol Behav Assess 2002;24:215-23.

24. Jenike MA. Obsessive-compulsive and related disorders: a hidden epidemic. N Engl J Med 1989;321:539-41. 